\title{
À l'encontre de la pensée unique roumaine : Vasile Ernu
}

Gina Puică

\section{(2) OpenEdition}

1 Journals

Édition électronique

URL : https://journals.openedition.org/cher/10531

DOI : 10.4000/cher.10531

ISSN : 2803-5992

Éditeur

Presses universitaires de Strasbourg

\section{Édition imprimée}

Date de publication : 1 décembre 2013

Pagination : 223-241

ISBN : 978-2-86820-560-5

ISSN : 1968-035X

\section{Référence électronique}

Gina Puică, «À l'encontre de la pensée unique roumaine : Vasile Ernu », reCHERches [En ligne], 11 | 2013, mis en ligne le 08 février 2022, consulté le 09 février 2022. URL : http://

journals.openedition.org/cher/10531; DOI : https://doi.org/10.4000/cher.10531

\section{(c) (i) (2) (2)}

Ce(tte) œuvre est mise à disposition selon les termes de la Licence Creative Commons Attribution Pas d'Utilisation Commerciale - Partage dans les Mêmes Conditions 4.0 International. 


\title{
À l'encontre de la pensée unique roumaine: Vasile Ernu
}

\author{
GINA PUIČ \\ Université de Strasbourg \\ Université « ştefan cel Mare » de Suceava - Roumanie
}

\section{Une identité complexe}

Le nom de la Moldavie vient rarement sous la plume de Vasile Ernu, bien qu'il soit né sur ce territoire (République Socialiste Soviétique de Moldavie) en 1971. La simple lecture de ses notices biographiques, qui commencent systématiquement par la phrase: «Vasile Ernu est né en URSS en 1971 », est une preuve de cette présence-absence.

En effet, ce n'est pas la Moldavie que l'écrivain considère comme étant sa patrie ni même la Roumanie, son pays d'adoption. La patrie de Vasile Ernu, c'est l'URSS - un impressionnant espace-temps vécu sous la perestroïka et idéalisé comme tout espace-temps de l'enfance. Mais s'y ajoute pour l'écrivain qu'est devenu entre-temps le jeune enfant de l'«Empire», la dominante «culturelle» de ce vaste territoire et son grandiose auto-démantèlement.

Rien de plus naturel pour Vasile Ernu que de bâtir, sur cette "patrie impériale » effondrée, l'espace d'appartenance de son être-écrivain, et même d'en faire sa raison d'être écrivain, étant bien connu qu'il n'y a rien de meilleur qu'une patrie perdue, exigeant d'être récupérée à travers l'écriture, pour nourrir un univers scriptural. Surtout Vasile Ernu voit large:

Que je le veuille ou non, je suis un enfant de l'Empire, né quelque part dans le Sud, à la périphérie. L'Empire - ceci est valable ailleurs que dans l'Empire russe ou soviétique - pose des problèmes liés à la définition d'un certain type d'identité. Il existe, certes, une identité ethnique, nationale, linguistique, géographique et religieuse à laquelle on appartient mais il y a encore quelque 
chose qui transcende tout cela. L'identité impériale vous donne quelque chose de plus que ces identités ou plutôt les réunit et leur imprime un sens qui va bien au-delà. L'appartenance à un empire vous donne un sens pardelà les identités nationales, religieuses et linguistiques, celles-ci devenant secondaires (Ernu ${ }^{1}, 2010$ b $^{2}$ : 101).

Ce fragment est tiré d'un texte bref et éclairant ( Cum străinătatea a devenit noua mea Patrie» [Comment l'étranger est devenu ma nouvelle Patrie]) publié dans Prima mea călătorie în străinătate, un ouvrage collectif dans lequel une trentaine de jeunes écrivains roumains évoquent leur premier voyage à l'étranger. Vasile Ernu trouve ainsi une nouvelle occasion de présenter son parcours, et de déployer en quelques pages les plis de son identité multiple, en présentant la Roumanie comme un pays étranger devenu sa nouvelle patrie mais non sans que ce passage ne lui ait posé quelques problèmes.

\section{La Roumanie - pays étranger plus que nouvelle Patrie?}

Vasile Ernu vit en Roumanie depuis le début des années 1990. Après avoir fait des études universitaires à Iaşi et à Cluj, il s'est installé à Bucarest où il est depuis environ le début des années 2000 une présence très active dans la vie culturelle. Collaborateur de plusieurs revues, dont certaines d'avant-garde, il a aussi, depuis quelques années, magnifiquement intégré la littérature de Roumanie. Et pourtant ce pays reste pour lui une patrie que, vingt ans après, il est encore et toujours en train d'acquérir, de s'approprier: donc une patrie encore vaguement attendue.

Une forme de gai désespoir se donne à lire sous la plume de l'écrivain quand il évoque le caractère relatif de son intégration roumaine. Il a beau maîtriser la langue, les coutumes, la culture de la nouvelle Patrie, il n'en ressent pas moins celle-ci comme «un corps étranger et artificiel» (Ernu, 2010 b: 106). Comme la langue maternelle, la patrie s'approprie pendant

1 Toutes les citations tirées de l'œuvre de Vasile Ernu (qui n'est pas traduite en français), présentes dans cet article nous appartiennent.

2 Étant donné que notre article renvoie à la dernière édition (2010) de Născut în URSS, et que nous citons également l'article "Cum străinătatea a devenit noua mea Patrie», également publié en 2010, afin d'éviter toute confusion, dans nos références, nous accolerons à l'année de publication les lettres $a$ (pour Născut în URSS), respectivement $b$ (pour «Cum străinătatea a devenit noua mea patrie»). Quant au livre Ceea ce ne desparte. Epistolarul de la Hanul lui Manuc, publié aussi en 2010, étant donné qu'il a deux auteurs, et qu'aucune confusion ne peut donc être faite, nous nous limiterons à noter l'année 2010 . 
l'enfance - telle est la conclusion, sur ce point, de Vasile Ernu, qui insiste encore sur la question du temps qui se mêle à celle des lieux. Comme tout "enfant de l'Empire», éduqué "dans une grande utopie», il se déclare inapte à vivre le temps de la même façon que ses nouveaux compatriotes. Il a besoin, lui, d'un «sens majeur qui unifie et non point fragmente la vie» (ibid.).

De ce fait, il n'est guère étonnant qu'il ait ressenti son déplacement géographique vers la Roumanie ainsi que son installation dans la nouvelle patrie, comme un déplacement éprouvant dans le temps aussi. Les années grises de la transition roumaine qui succédèrent pour le jeune Vasile à la flamboyante période de la perestroïka, qu'il venait de traverser et à laquelle il reste affectivement très attaché, furent pour lui des années d'extrême épuisement. Un vrai exil, que nous pourrions interpréter comme une traversée du désert avec la promesse d'une vraie transfiguration à la clef. Une épreuve initiatique l'ayant mis sur la voie royale propre à un écrivain très respecté dans le cadre des Lettres roumaines actuelles.

La question de son appartenance et de son identité reste donc problématique. Mais ce trait n'est-il pas une sorte de condition sine qua non de tout parcours intellectuel abouti? En tout cas, l'«hérétique» Vasile Ernu, comme il aime à se décrire, ne semble s'accommoder d'aucun positionnement définitif, précis ou juste banal. Et il est peu probable qu'un ancrage plus marqué dans l'identité moldave ou roumaine lui soit d'un grand secours. C'est ce qui fait aussi son originalité, à l'heure où bon nombre d'écrivains et d'intellectuels de la République de Moldavie actuelle gomment jusqu'à l'extrême leur différence spécifique, se laissant influencer et assimiler par les milieux conservateurs de Roumanie.

En plus, le fait de proclamer haut et fort son identité «impériale» procure à Vasile Ernu une liberté d'expression qui lui permet de scruter de manière très critique et originale les dérives et les lâchetés de tous ordres de l'intelligentsia et des politiques roumains du présent.

\section{Un homo sovieticus éclairé}

La perspective dans laquelle Vasile Ernu affirme se situer lorsqu'il est à l'ouvrage est celle d'un "homucus», d'un homo sovieticus éclairé, qui regarde lucidement le monde disparu, auquel il s'identifie en partie, depuis le monde qui lui a succédé et dans lequel il vit. Or, le monde slave, et surtout le régime soviétique représentent aujourd'hui une sorte d'altérité absolue pour les Roumains: une altérité à rejeter et non point à cultiver. En cela, 
Vasile Ernu apporte, dans une Roumanie où le débat est assez consensuel, une touche extrêmement personnelle. Et cette posture, vue par plus d'un comme étant «irresponsable», offre des avantages intellectuels stimulants, sinon vertigineux, dont notamment la possibilité de critiquer le monde contemporain à travers une critique des critiques qui sont actuellement généralement formulées à l'endroit du passé.

Ce rapport au passé n'est certes pas dépourvu de nostalgie, ni d'une audace particulière:

En regardant derrière moi, je me rends compte qu'avec la disparition de l'Union Soviétique et du communisme, quelque chose s'est perdu. Peutêtre est-ce un certain pathos, une certaine manière de voir les choses et de vivre la vie de tous les jours, peut-être est-ce l'enthousiasme de croire encore aux idéaux, peut-être est-ce enfin une certaine manière de souffrir. Je suis incapable d'identifier la perte, mais je vis la tristesse de cette perte. Je crois non sans une certaine conviction que quelque chose d'essentiel et de significatif de notre expérience humaine est définitivement perdu. Mais cette perte ne peut être ni remplacée ni réhabilitée. Je crois en revanche qu'avec encore un peu d'effort, cette perte peut être comprise. (Ernu, 2010a: 9)

Et Vasile Ernu poursuit:

De temps à autre j'ai envie d'acheter un billet pour l'URSS, mais chaque fois je dois me rappeler que cela ne se vend plus. Nul train, nul avion et nul chemin ne mènent plus en URSS. [...] La seule façon de visiter mon pays c'est de faire travailler la mémoire. (Ernu, 2010a: 9-10)

Ce n'est pas d'une nostalgie naïve qu'il s'agit ici car Vasile Ernu ne se contente pas de simplement faire retour sur le passé et de le ramener tel quel vers le présent. Une nostalgie active le porte, qui le rend capable d'interroger le passé depuis le présent et avec les moyens du présent, afin de mieux remettre en question le présent lui-même à travers les leçons du passé, dans un va-et-vient des plus stimulants.

D'ailleurs, son premier livre, Născut în URSS comme le suivant, sont rédigés dans une double perspective. Il y a d'abord l'expérience directe, mais retravaillée par la mémoire, de cet homo sovieticus, de cet homucus, que Vasile Ernu considère être et puis il y a aussi un bagage culturel, idéologique très diversifié qu'il exploite et met à l'œuvre 3 .

Une affaire d'intellect plus que d'affect, car ce qui impressionne, motive, fascine et trouble Vasile Ernu c'est cette URSS vue comme construction

3 Parmi les sources livresques de Vasile Ernu comptent Slavoj Žižek, Giorgio Agamben, Walter Benjamin, Carl Schmitt, Michel Foucault (Ernu, 2009: 309). 
culturelle, opposée aux constructions naturelles, l'URSS en tant que "projet politique utopique de la modernité» le plus important que notre auteur décrit comme l'«écriture politique la plus grandiose du $\mathrm{xx}^{\mathrm{e}}$ siècle» (Ernu, 2010a: 7), où «ce n'était pas l'art qui imitait la vie, mais la vie qui devenait de l'art» (ibid.).

\section{Né à la littérature sous le signe de l'URSS perdue}

Le livre de début de Vasile Ernu, Născut în URSS4, paraît en 2006 aux éditions Polirom, comme la plupart de ses livres. Envisagé au départ comme projet collectif, il fut finalement assumé par seul Vasile Ernu, car la plupart de ses amis initialement partants s'étaient entre-temps dispersés de par le monde. Concrétisé dans un recueil de textes assez brefs, et hybrides (pouvant être rattachés plus ou moins à des genres comme l'essai, les mémoires, le journal intime et le reportage), le livre porte sur des sujets et des aspects très variés de la vie courante en URSS, à l'aide desquels l'écrivain constitue un kaléidoscope vivant de la vie soviétique, allant des grandes figures révolutionnaires au sexe et à l'alcool, en passant par les héros de romans et de films, la vie communautaire dans les appartements nommés Kommunalka, la propagande, l'intelligentsia et la dissidence.

Très bien accueilli, ayant reçu plusieurs prix importants, le livre bénéficie déjà de deux rééditions, et de plusieurs traductions à l'étranger. Mais il ne manqua pas de faire naître quelques polémiques en Roumanie, car l'auteur apporte une vision qui a quelque chose de dérangeant: qui d'autre oserait aujourd'hui en Roumanie, où le sentiment anti-russe et anti-soviétique est très puissant, comme nous le suggérions, s'attendrir sur l'Union Soviétique, voire déplorer ne serait-ce qu'un peu sa disparition?

Mais disons-le nettement: Vasile Ernu n'est pas l'admirateur béat de l'URSS, et il est loin d'éprouver de la nostalgie à l'état pur. Si beaucoup de personnes, y compris Sorin Antohi, l'auteur de la postface de son premier livre, crurent distinguer plus de nostalgie qu'il n'y en a dans le texte de Vasile Ernu, ceci s'explique par le sentiment puissamment anticommuniste qui se manifeste de nos jours en Roumanie, qui transforme toute référence non vigoureusement critique à l'égard du monde russe/soviétique en sujet tabou.

4 Le livre en est déjà à sa troisième édition: après une $1^{\text {re }}$ édition en 2006, s'ensuivirent les $2^{e}$ et $3^{e}$ éditions en 2007 , respectivement 2010. C'est à cette dernière édition que nous renvoyons. 
Les milieux intellectuels conservateurs de Roumanie ont, en effet, des difficultés à comprendre que l'on peut thématiquement s'attacher au monde soviétique disparu pour comprendre et mieux faire comprendre, depuis ce passé et ce monde disparu, le présent de la Roumanie postcommuniste, comme de l'Occident capitaliste en général. Donc, en faisant de l'URSS la matière de ses livres, Vasile Ernu marque ainsi sa différence par rapport aux milieux roumains qu'il croise, dont la préoccupation essentielle semble être invariablement la dénonciation des oppressions communistes. Et, ce faisant, il montre aussi comment ce positionnement quasi-général, à l'encontre duquel il se place, n'est qu'un travail sur commande, voire une imposture commode, dont le seul bénéfice est de mettre pitoyablement les intellectuels à l'abri de tout risque.

Vasile Ernu aurait pu, après la publication de son premier livre, se tourner vers d'autres thèmes, mais son cheminement d'écrivain entamé avec Născut in URSS se poursuit avec la rédaction d'un livre beaucoup plus complexe et encore plus audacieux littérairement et idéologiquement parlant: Ultimii eretici ai imperiului [Les derniers hérétiques de l'Empire], où la part du souvenir est moins directement détectable que dans son premier livre. Présenté sous la forme d'un roman épistolaire entre un mystérieux A.I., dénommé aussi le "Grand Instigateur", décrit comme un ancien terroriste ayant essayé d'assassiner Staline et ayant réchappé à toutes les catastrophes, et Vassily Andreevich, double évident de l'auteur, le livre s'affirme toujours comme un «ensemble d'histoires, d'idées et d'obsessions auxquelles l'auteur s'est vu confronter [dans la Roumanie] de la transition [postcommuniste] » (Ernu, 2009: 309), comme l'écrit Vasile Ernu dans une note finale du livre.

\section{Idées hérétiques}

Le cheminement de Vasile Ernu que nous venons de présenter est une preuve du caractère "hérétique» de ses idées, qui se placent à l'encontre de ce qui est communément admis actuellement en Roumanie - espace dans lequel il évolue, pour lequel il écrit, et qu'il ambitionne sans doute de remodeler. Vasile Ernu en est le premier conscient, car il fait de la démarche hérétique une forme de programme. Il définit l'hérésie comme étant

une croyance qui se trouve en contradiction avec les dogmes officiels. L'hérésie est une forme radicale, minoritaire, marginale de critiquer quelque chose d'officiel et de majoritaire. C'est la périphérie de la croyance, la périphérie de l'Empire, quel que soit cet Empire. Et je sais très bien que l'hérésie est presque toujours condamnée et punie. (Ernu, 2009: 210-211) 


\section{Les nostalgies interdites}

Participent d'une forme (légère) d'hérésie, ce que nous pouvons présenter comme étant les nostalgies interdites dans la Roumanie d'aujourd'hui, et que Vasile Ernu cultive avec délice et non sans dénoncer par la même occasion les positions opportunistes de ses nouveaux conationaux.

a) De l'existence historique de l'URSS...

À cette enseigne, il faut mettre d'abord la nostalgie pour l'URSS ellemême, et sa géographie particulièrement grandiose, dont «les distances se mesurent en temps» (Ernu, 2009: 11) - ainsi apprend-on que la distance entre Chişinău et Moscou est de 36 heures de train! Vasile Ernu se montre durablement fasciné par ce qu'il voit comme étant «le plus grand projet politique de la modernité» (Ernu, 2010a: 7); par la performance d'une méga-construction politique:

Nous y avons tous vécu la plus grandiose écriture politique du $\mathrm{xx}^{\mathrm{e}}$ siècle, aux élans héroïques, aux efforts inhumains, aux tragédies terribles, aux victoires et défaites sanglantes. Nous y avons vécu une écriture qui nous a permis à tous d'être contemporains les uns des autres. Nos héros, les personnalités et les personnages, les mots et les choses, quelle que fût la période réelle où ils sont nés et où ils ont vécu, ont habité à nos côtés, dans la même kommunalka nommée URSS, en étant simultanément nos précurseurs et nos contemporains. (Ernu, 2010a: 7-8)

Associé au grand thème de l'existence passée de l'URSS (et de la nostalgie présente pour elle), il y a le thème diffus de l'utopie en tant que spectacle de la pensée, que Vasile Ernu récupère, engagé comme il est dans une réflexion idéologique très pointue, nourrie à des sources variées allant des écrivains et idéologues les plus classiques russes et occidentaux jusqu'aux penseurs anarchistes contemporains. Or, cette position n'est pas du goût des intellectuels les plus en vue de Roumanie, fort conservateurs, aux yeux desquels l'utopie est une invention quasi-diabolique, ou qui la dénoncent tout simplement comme étant une composante essentielle de la pensée de gauche. Et, comme le dit l'auteur par la voix de son porte-parole Vassily Andreevich, "L'intelligentsia roumaine n'a aucun appétit pour la pensée de gauche. La gauche a été et est encore traitée [ici] comme une pensée d'occupation» (Ernu, 2009: 29).

b) ...à la kommunalka, cette «micro-URSS »

Viennent, ensuite, des aspects très matériels de la société communiste soviétique pour lesquels la nostalgie de Vasile Ernu reste intacte et qui 
lui font écrire des textes sublimes, surtout dans Născut în URSS. La kommunalka, le type d'habitation commune, que l'écrivain désigne comme étant la «synthèse de la civilisation soviétique» (Ernu, 2010a: 43) en est un des plus éloquents. Résultat de la Révolution d'Octobre, la kommunalka était une grande maison bourgeoise transformée par oukase en habitation pour plusieurs familles ( $10 \mathrm{~m}^{2}$ distribués par locataire). Chaque famille y disposait d'une chambre, qui constituait l'espace privé de la maison, et avait accès aux espaces communs: le corridor, les WC, la cuisine, et la salle de bains (Ernu, 2010a: 43-44). L'espace privé fort rétréci, sinon absent, de ce type de logement ne semble pas un inconvénient pour l'homo sovieticus, éduqué par les idéologues de service dans l'esprit du partage total, et vite habitué, voire devenu dépendant d'un mode de vie fondé sur ce principe.

Vasile Ernu avertit tout lecteur profitant aujourd'hui des « plaines fertiles du Canaan capitaliste» (Ernu, $2010 \mathrm{a}: 44$ ), et qui se plairait à disserter sur la démarcation entre public et privé, à ne pas confondre la kommunalka avec l'un ou l'autre de ces espaces:

[...] si je devais revenir aux origines de l'humanité, je dirais que cette kommunalka est le Jardin de l'Eden, le lieu où se trouve l'arbre de la connaissance du bien et du mal, pour d'aucuns un enfer, pour d'autres un paradis. Et comme nous ne prisons pas l'opium des peuples, je vous dirai sincèrement que pour nous la kommunalka est le lieu de l'amour et de la haine, de l'amitié et des séparations, de la jalousie et de la tendresse, de la joie et de la tristesse ou, plus simplement dit, notre vie même. Comme dirait un chant soviétique: «Mon adresse ne comporte ni numéro, ni rue; mon adresse c'est l'Union Soviétique» - en d'autres termes, la kommunalka. (ibid.)

L'invention et surtout la réalité de la kommunalka surpassent les projets utopiques de Campanella, Owen et Fourier, Bacon et Huxley par ses transformations et celles subies par ses résidents, affirme même Vasile Ernu (ibid.).

Même s'il évoque essentiellement ses propres souvenirs, l'écrivain entend parler au nom de tous les ex-Soviétiques. Les souvenirs de sa tante Klava ouvrageant dans la cuisine commune telle un perpetuum mobile devient un emblème de toute vie menée dans une pièce semblable en ce temps-là. C'est dans cette pièce de la kommunalka que les amoureux soviétiques se donnaient rendez-vous autour d'un verre de vin rouge, et que les amis se disputaient autour d'une bouteille de vodka. Lieu où la musique était jouée et écoutée, où des plans étaient minutieusement bâtis, et des poèmes lus, où l'on bavardait, et méditait sur le sens de la vie, c'était en somme un endroit tenant lieu de «club, salon, cénacle», voire «le plus important laboratoire de 
mythes et de réalités» (Ernu, 2010a: 45). "Si on nous avait pris la cuisine, on nous aurait pris presque tout. Ils pouvaient tout nous prendre, sauf la cuisine. Notre vie, notre âme étaient devenues une partie de la cuisine, et la cuisine une partie de notre âme» (ibid.).

L'importance des relations de voisinage était, évidemment, capitale dans la kommunalka. Et cela devait avoir un revers. Comme pour cette femme soviétique partie aux USA et qui demanda une cuillerée de sel à un voisin, lequel jugea bon d'appeler la police!

[Cette femme] est morte quelques années plus tard, sans doute parce qu'aucun voisin capitaliste ne lui avait donné une cuillerée de sel. Ils n'ont pas compris que ce n'est pas de sel qu'elle avait besoin, car elle pouvait s'en acheter une tonne. Elle avait besoin [...] de cette cuillerée soviétique de sel dont on ne pouvait pas se passer dans la kommunalka et qui y jouait le rôle d'une bulle d'oxygène. (Ernu, 2010a: 45-46)

\section{La remise en cause des idées reçues roumaines}

Les textes de Vasile Ernu ont trouvé leur public autant dans l'ex-URSS que dans d'autres espaces. Mais s'il est un endroit où la présence de Vasile Ernu s'avère incontournable, c'est bien la Roumanie, où l'écrivain est véritablement parvenu à remplir un espace vide. Le fait qu'il s'y soit taillé une place bien à lui est de la meilleure utilité pour l'enrichissement du débat dans ce pays, passablement monochrome dans sa vision dominante, et très binaire dans le partage que cette dernière induit. C'est pour cette raison que l'apport intellectuel, voire idéologique, de Vasile Ernu donne à son écriture toute sa raison d'être.

En effet, la perspective décalée de Vasile Ernu qui tente de comprendre le contemporain à travers l'expérience soviétique, et celle-ci à travers les données du présent occidental et capitaliste, au profit du présent mais aussi de l'histoire des idées, comptent parmi les rares prises de position qui se soustraient à l'ordre dominant, se plaçant à rebours des discours des intellectuels roumains les plus en vue.

a) Misère du discours anticommuniste post-1989

Le point de litige le plus remarquable qui oppose Vasile Ernu et l'establishment intellectuel roumain reste le positionnement par rapport au communisme. Depuis plus de deux décennies, la fine fleur de l'intelligentsia roumaine ne cesse d'accabler l'époque communiste. Si cette position est tenable et bien justifiée, ce qui interpelle Vasile Ernu, c'est que ce 
positionnement, par sa persistance, soit devenu l'unique raison d'engagement publique à l'heure où l'affaire est close, et qu'il ait accaparé toute l'énergie de ces intellectuels, qui ne voient plus les problèmes réels ni les vrais combats d'avenir. La façon dont cette intelligentsia se sert du discours anticommuniste est donc une «imposture» pour Vasile Ernu:

Même si le discours anticommuniste est porteur de nombreuses vérités, il faut s'apercevoir que la proclamation publique de ces vérités est fondée sur l'imposture, car elles sont prononcées et assumées à une époque où elles ne peuvent plus remplir de fonction critique, et où elles ont même vocation à détourner la finalité, le rôle et la problématique du discours critique lui-même. Aujourd'hui, au lieu de questionner, nous condamnons et dénonçons ${ }^{5}$; au lieu de voir les effets de l'ancienne idéologie sur la société actuelle, nous forgeons une contre-idéologie. À l'idéologie communiste disparue s'oppose une nouvelle idéologie: l'anticommunisme. C'est une idéologie qui ne comporte pas de risque, juste du profit. La grande tragédie de l'intelligentsia roumaine consiste en cela qu'à travers ce type de gestes, elle perd sa légitimité, et avec les discussions autour du communisme-postcommunisme, tout se réduit en fait à un seul problème essentiel: l'incapacité des intellectuels à prendre une position critique face au Pouvoir et à l'idéologie dominante du moment, quel que soit ce moment. (Ernu, 2009: 30)

L'écrivain considère que la situation est d'autant plus sujette à critiques qu'il décèle dans ce type de prise de position chez les intellectuels roumains ("petits enfants de Dracula», 2009: 25) un souci constant de préservation de leurs propres intérêts et autres privilèges. Et le mot pour caractériser ces personnalités publiques fut vite trouvé: "priviligentsia».

Ce que reproche aussi Vasile Ernu à ces intellectuels et autres politiques, outre de prendre une posture pour eux favorable, c'est de ne pas savoir faire accompagner leur critique-condamnation d'une plate-forme de réflexion et de débat ni d'une structure capable de faire justice aux victimes (Ernu, 2009: 86). Il ne peut que s'apercevoir donc du caractère «abstrait» de leur condamnation, de leur incapacité à assumer eux-mêmes ce passé dénoncé,

5 L'auteur fait notamment référence au Rapport de la Commission Présidentielle pour l'Analyse de la Dictature Communiste en Roumanie, réalisée par une équipe de chercheurs sous la direction de Vladimir Tismăneanu, rapport sur lequel se fonda le Président Traian Basescu, en décembre 2006, pour condamner solennellement devant le Parlement la période communiste, en la déclarant comme «illégitime» et «criminelle». Vasile Ernu dirigera, aux côtés de Costi Rogozanu, Ciprian Șiulea et Ovidiu Țichindeleanu, un ouvrage venant en réponse à ce rapport: Iluzia anticomunismului. Lecturi critice ale Raportului Tismăneanu (Editions Cartier, Chișinău, 2008). 
et de l'inutilité pour la vie de l'esprit, voire du ridicule de ce genre de prise de parole publique (Ernu, 2009: 85).

[...] que cela leur plaise ou non, [le communisme] ne peut être effacé, «condamné» et déclaré comme «illégitime». On ne peut pas émettre un décret par lequel annoncer que ce qui s'est passé pendant 45 ans est «illégitime» et «criminel». C'est ridicule d'être illégitime durant toute une moitié du $\mathrm{xx}^{\mathrm{e}}$ siècle. [...] Le fait est qu'ici on ne veut ni comprendre ni assumer. L'histoire est pour la plupart de nos intellectuels un «esprit », et, eux, ils se prennent pour des chamans. Ils s'adonnent à des rituels d'exorcisation, éloignent le «mauvais esprit», le «mal», condamnent la «foudre» et l'«épidémie», et amènent la "pluie» et le «bon esprit». La majeure partie de notre intelligentsia est devenue un groupe de petits trafiquants et de faussaires d'idoles - idoles de la cité, de la caverne, de la place publique, ou du théâtre, au choix. (Ernu, 2009: 84-85)

Pour évoquer ce même manque de responsabilisation face à l'histoire récente, dans un livre écrit à deux mains avec Bogdan-Alexandru Stănescu, Vasile Ernu parle d'une histoire roumaine relevant du domaine «météorologique» (Ernu, Stănescu, 2010: 158) - une histoire «naturelle», et non point "culturelle», qui «nous arrive», «se décide en notre absence», "presque par-delà toute volonté» (ibid.). Il revient pour l'occasion sur ces «intellectuels-sorciers» et leur approche de l'histoire à «tendance animiste» : "[...] si la foudre a frappé ta maison, l'intellectuel-sorcier jette l'anathème sur la foudre; si la sécheresse sévit, les intellectuels sortent avec les icônes sur les champs pour quémander la clémence divine» (Ernu, Stănescu, 2010: 159).

Déplorant par la même occasion l'absence de «débats sérieux» dans la vie publique - comprenant par là, entre autres, «des textes qui puissent devenir des critiques dévastatrices de notre temps» (Ernu, Stănescu, 2010: 166) ainsi que l'absence de créations relevant de l'utopie et de la contre-utopie dans toute l'histoire de la littérature roumaine ${ }^{6}$, Vasile Ernu remarque le fait que les intellectuels y travaillent uniquement sur «commande d'État» (Ernu, Stănescu, 2010:159). «Si une partie de l'Histoire ne nous convient pas, nous la déclarons illégale, et la condamnons. C'est-à-dire nous l'instrumentalisons» (ibid.). Cette instrumentalisation de l'histoire transformerait cette dernière en marchandise, selon Vasile Ernu.

6 La littérature roumaine préfère, selon Vasile Ernu, les «animaux non-politiques: papillons, chats, anges, huppes, etc. Ce sont des animaux adorables qui méritent d'entrer dans la littérature, sauf que les <démons> de la littérature sont ailleurs » (Ernu, Stănescu, 2010: 159). 
b) Mais où est passé le politique?

Au-delà de toute condamnation officielle du communisme, il est de notoriété qu'il y a un double regard porté sur l'époque communiste dans la Roumanie d'aujourd'hui. On dénonce violemment sa première période, celle de Gheorghiu-Dej (1947-1965), dite "stalinienne», et on considère avec clémence celle, nationale-communiste, de Nicolae Ceaușescu (1965-1989). Déjà en partie dénoncée et condamnée comme «stalinienne» durant la période «ceauşiste $»^{7}$, la première étape du communisme roumain continue aujourd'hui d'être accusée de tous les crimes, alors que l'époque immédiatement postérieure est généralement jugée, par la plupart des idéologues de circonstance, comme ayant été relativement dépourvue de violences (alors qu'elle fut tout aussi violente, à cette différence près qu'elle se donnait d'autres cibles et d'autres moyens, car elle s'ancrait dans un contexte différent).

Si les critiques à l'égard de l'époque de Gheorghiu-Dej sont justifiées, ce qui gêne donc dans cette approche, c'est le fait que la différence de traitement entre les deux périodes contribue à sauver de facto le communisme nationaliste roumain. En effet, avec Ceauşescu le communisme internationaliste («Communistes de tous les pays, unissez-vous!») bascule en national-communisme (détachement feint ou réel de Moscou, indépendance par rapport à la politique d'ensemble du Pacte de Varsovie, et a contrario une attention particulière portée à la réécriture de l'histoire nationale, et à tous faits et gestes pouvant magnifier la Patrie). Ainsi, quand Vasile Ernu écrit - ironiquement, mais pas trop - que l'époque «ceauşiste» fut plus pernicieuse que l'époque précédente, brise-t-il un tabou et peut-il blesser l'orgueil national de certains Roumains.

Pour nous en tenir à la littérature et aux effets du national-communisme sur elle, un seul exemple : il est acquis, en Roumanie, qu'en ce domaine le second communisme roumain offrit toutes les libertés de création, après une décennie de réalisme socialiste (proletkultisme) au pouvoir, entendant par là que les écrivains pouvaient à nouveau librement redéployer leur énergie créatrice hors du domaine socio-politique auquel ils y étaient tenus jusque-là, au profit de la poésie, et d'une redécouverte conséquente des espaces intérieurs, mythiques, magiques, et autres, qui permettaient que le formalisme l'emportât sur l'idéologique. D'habitude, on voit en cela une

7 L'«auto-critique» et le meurtre (réel ou symbolique) des pères étaient, par ailleurs, l'une des caractéristiques du communisme. 
liberté d'expression recouvrée et une normalisation de la vie littéraire sous le communisme.

Vasile Ernu, lui, renverse ce constat, et remarque, au contraire, combien cette littérature (et plus largement cette culture) sous Ceauşescu se fourvoyait dans sa fuite hors du politique, combien la fameuse "résistance par la culture», tant vantée et présentée comme modèle de la résistance roumaine sous le communisme, n'était qu'une fable - et même une fable dangereuse:

Le «retrait» général de la sphère du politique signifie participation directe à son renforcement. Le renoncement à la critique, au doute, à la chicane indirecte suppose de petits hommages rendus au pouvoir. Ceauşescu n'a pas existé, c'est nous qui l'avons produit, ceux qui l'avons acclamé comme ceux qui nous sommes tus dans notre soumission docile. Ceauşescu est une espèce de projection collective, une sorte d'écriture collective que nous avons conçue tous ensemble. Même les quelques dissidents ont contribué à la production de l'époque Ceauşescu, car ils l'ont légitimée et lui ont donné un contour, une image plus claire, fût-elle négative. Ils ont montré, à leur insu, que «ce mal» existe ici et maintenant, en dehors de nous. (Ernu, 2009: 87)

Et Vasile Ernu ajoute tout de suite après et sans ambages: "Ceauşescu, hélas! reste le produit culturel et politique le plus important de la Roumanie moderne» (ibid.). Quelques pages plus loin, il revient sur cette question, et l'auteur enchaîne, par la voix de son double Vassily Andreevich, et en référence à l'œuvre de Varlam Chalamov (auteur des Récits de la Kolyma):

L'absence du politique rend possible l'apparition du système totalitaire. [La théorie de Chalamov] renverse la grille d'interprétation de l'establishment idéologique [roumain] actuel qui considère que c'est l'excès de politique qui a créé ce régime, et qui, indirectement, nous invite à la prudence en matière de politique. (Ernu, 2009: 98)

La «résistance par la culture» laisse donc la voie libre aux régimes totalitaires, et Vassily Andreevich approuve son correspondant A.I, quand celui-ci affirme que «Staline et Ceaușescu sont des produits culturels, des métaphores, des concepts élaborés par nos cerveaux, les résultats de nos agissements» (Ernu, 2009: 103). Enfin, par l'intermédiaire de A.I., encore, Vasile Ernu rappelle que la responsabilité pour «l'âge d'or» communiste roumain ne devrait pas être rejetée sur les Soviétiques (selon la tendance du moment), car il est le produit de l'époque qui a suivi le retrait des troupes soviétiques de Roumanie (1964). Et l'auteur de déplorer alors, par le biais des lettres que s'échangent ses personnages, le départ anticipé de l'occupant, dont la présence prolongée aurait empêché en Roumanie la création par 
la suite d'un dictateur local et, a contrario, aurait encouragé (peut-être) la naissance d'un discours publique énergiquement dirigé contre l'occupant ${ }^{8}$.

\section{Les hérésies absolues}

Enfin, et non en dernier lieu, les textes de Vasile Ernu empruntent presque par endroits la voie de l'hérésie absolue.

a) Capitalisme versus Communisme ou Capitalisme égale Communisme?

Selon Vasile Ernu, les différences entre ces deux systèmes politiques que l'on présente habituellement comme irréconciliables sont de pure apparence, tandis que leur ressemblance est essentielle, car les deux se fonderaient sur la répression. Si le communisme porte à la base la répression politique; le capitalisme s'appuie sur la répression économique (Ernu, 2009: 135). À la terreur politique mise en œuvre par le communisme soviétique correspond donc, en une parfaite symétrie, la terreur économique propre au capitalisme.

Pour que la remarque n'ait pas l'air d'une simple boutade, Vasile Ernu étaie son affirmation par force exemples. La question du temps en est un. Si les Soviétiques et autres Est-européens disposaient du temps long communiste qu'ils meublaient tant bien que mal, dans le monde capitaliste on ne cesse de déplorer le manque de temps. Et ne pas disposer de son temps c'est précisément ne pas être libre, ne pas agir en vertu de son projet personnel, mais se voir bâtir un projet de fortune, soumis aux restrictions du système (Ernu, 2009: 136). L'institution bancaire qui régit la vie de tous les individus vivant dans le monde capitaliste est un autre exemple de l'intrusion dans l'existence humaine de règles agencées en un puissant «système de contrôle et de répression» (Ernu, 2009: 137) et en cela contredisant la liberté tant clamée. De même que l'institution de l'Assurance, parfait exemple du besoin de sécurité ressenti par l'individu (Ernu, 2009: 138).

Mais la liberté n'est-elle pas au fond impossible, ou - pis - insupportable? Inconciliable avec l'idée de sécurité (donc de stabilité), elle ne peut dans la

8 À des intellectuels et politiques roumains qui refusent obstinément d'assumer le communisme, le considérant comme un pur produit de l'occupation soviétique, l'auteur écriten toute clarté: «Des pays comme la Roumanie, l'Albanie et la Bulgarie, d'où les troupes soviétiques se sont retirées [plus tôt], ont été peut-être les plus dociles et ont développé les plus durs systèmes communistes, tandis que des pays comme la Hongrie ou la Tchécoslovaquie, où les troupes soviétiques sont restées, ont vu naître les groupes d'intellectuels les plus libéraux et les structures politiques les plus dynamiques ayant conduit à des changements significatifs» (Ernu, 2009: 108). 
réalité des faits qu'être abandonnée, mais seuls les ex-communistes s'en rendent vraiment compte.

Vasile Ernu remarque aussi que la soumission au système bureaucratique dans le monde capitaliste rend impossible l'existence des héros, ces êtres si adulés dans l'espace russe. Comment devenir héros, se demande-t-il, si avant toute initiative il faut déposer une demande à la Mairie? Ici Vasile Ernu invoque ironiquement Hegel: «Si je me souviens bien, celui qui a le premier affirmé cela est ce philosophe allemand qui savait tout et que nous appelions Ghegheli: le système économique moderne et l'État de droit ne vous permettent plus de devenir des héros» (Ernu, 2009: 139). À moins d'être Hitler, Staline ou autre Ceaușescu, «les seuls hommes libres dans le monde moderne, parce qu'ils ont pu mener à bien leur propre projet de vie». Le double de Vasile Ernu affirme citer sur ce point un «ami penseur qui aime expérimenter toutes sortes d'idées", et qui ajoute que les gens vivant dans le régime capitaliste sont tout aussi soumis que les sujets communistes sans pour autant être contemporains de ces "grands hommes libres» (Ernu, 2009: 138-140).

Le rapport des masses aux "grands hommes libres» serait d'ailleurs lui aussi révélateur de la fausse contradiction entre les deux systèmes. Si dans le communisme, on pouvait s'en prendre à tout le monde et en toute impunité, à son chef compris, on ne pouvait en revanche s'attaquer au chef suprême. Dans le système capitaliste, on peut injurier tant que l'on veut sur la place publique le chef de l'État, mais on ne peut émettre la moindre critique à l'égard du chef de l'entreprise et d'autres petits chefs, sous peine de perdre son travail. Vassily Andreevich reproduit ici l'observation du personnage nommé «l'Enthousiaste», qui, renvoyant dos à dos capitalisme et communisme, affirme que dans le capitalisme, la démocratie se trouve en haut de l'échelle et le totalitarisme à sa base, alors que dans l'autre, on assisterait à un dispositif renversé.

Ce qui retient l'attention de Vasile Ernu et contribue à sa fascination (critique toutefois) pour le monde soviétique, c'est que le projet communiste a tout fait pour subordonner complètement l'économique au politique (contrairement au capitalisme). Or, quand le politique l'emporte, c'est la langue qui est souveraine. «Le politique se fonde sur des mots, des phrases, des décrets, des arguments, des programmes, c'est-à-dire sur le langage, tandis que l'économique se fonde sur des chiffres, sur l'argent» (Ernu, 2009: 76). Se faisant l'écho du personnage désigné sous le nom de «l'Esthète», Vassily Andreevich, écrit à A.I. que la Révolution d'Octobre fut un 
«linguistic turn» qui opéra le transfert de l'ensemble de la société du milieu financier, où elle se trouvait déjà, vers le milieu linguistique (ibid.).

Comment un intellectuel - de l'ex-URSS, qui plus est - ne regretteraitil pas le bon vieux temps où le seul groupe à avoir de l'influence était celui des intellectuels, semble se demander A. I. La langue étant l'instrument du pouvoir étatique, l'intellectuel communiste avait la possibilité de «légitimer et délégitimer le Pouvoir» (Ernu, 2009: 77), alors que l'intellectuel capitaliste voit ses discours réduits à intégrer des techniques quelconques, avant d'être récupérés par le marché. Ceci explique pourquoi les seuls dissidents et ennemis du système capitaliste sont aujourd'hui les terroristes, qui, ayant compris qu'il n'y a pas de dialogue possible par le truchement de la parole, invitent à un dialogue autrement violent avec le Pouvoir dominant (Ernu, 2009: 80-81, 192-193).

La question de la vraie-fausse différence entre communisme et capitalisme analysée, A. I. dénie par ailleurs assez formellement les tentatives récurrentes de mettre dans un même paradigme communisme et fascisme, et il considère, au contraire, que c'est plutôt le capitalisme qui serait plus proche du fascisme! La logique métrique, mathématique, calculatrice du système capitaliste renverraient aux mesurages de la biologie et autre génétique fascistes:

Dans l'idéologie et les sciences fascistes, le «juif» est une entité «biologique» qui peut être mesurée et définie avec exactitude et qui se distingue de l'«aryen ». Ainsi, l'entité «juif» est-elle pour l'«aryen pur» un produit «biologique», non pas un culturel. [...] Les camps de concentration fascistes ont pour but le contrôle total sur la «nature», et donc la liquidation des natures «impures»: juif, tzigane, handicapé, homosexuel, noir, communiste, etc. (Ernu, 2009: 78)

Dans l'idéologie communiste, en revanche, l'ennemi est toujours culturel. On ne naît pas, on devient un «ennemi du peuple». Et le personnage de Vasile Ernu de faire un lien inattendu entre système communiste et... religion! Le Goulag, construit pour «rééduquer», et non pas pour exterminer, laisserait la chance au rachat, constituerait une sorte de purgatoire9. Néanmoins, A. I. est non moins convaincu, son cas personnel à l'appui, de ce que cette "chance» offerte par le Goulag était automatiquement créatrice d'une souffrance

9 Et il ajoute: «Les intellectuels staliniens sont plus près des pères de l'Église chrétienne, de la patristique, des intellectuels médiévaux qui produisaient des textes pour bâtir la <cité de Dieu $>$. Le $<$ Tchevengour $>$ de Platonov, cette $<$ cité du Soleil $>$ est très proche de la $<$ cité de Dieu>». (Ernu, 2009: 78). A contrario, le capitalisme est jugé comme étant une "religion païenne» qui ne promet pas de salut, et où la divinité a été déplacée dans l'univers terrestre afin de produire, après avoir été vidée de transcendance (Ernu, 2009: 122). 
«indéfinie, illimitée, car elle ne tenait pas d'une limite temporelle, de règles claires, étant affaire de perpétuelle interprétation» (Ernu, 2009: 79).

b) Goulag et Glamour Goulag

Ancien détenu du Goulag, A.I. écrit à Vassily Andreevich en être nostalgique, et, comme pour choquer son correspondant, il affirme même, en généralisant son propos: "plus le système, le temps et le lieu sont restrictifs, concentrationnaires, durs, plus les souvenirs sont doux» (Ernu, 2009: 201 $)^{10}$. "Douleur plaisante», le souvenir du Goulag est comparé à un souvenir d'enfance attendrissant, ou encore à un amour de jeunesse mêlant plaisir et douleur, et qui dans certains cas n'a pas su remplacer efficacement et heureusement la réalité du Goulag. Pour preuve ces co-détenus invoqués par A.I. et qui, une fois libérés, se sont suicidés ne pouvant plus supporter une vie normale, ainsi que les intellectuels dissidents passés à l'Ouest, qui ne trouvèrent nulle part le bonheur promis et qui s'enfermèrent dans l'amertume et la volonté de retourner chez eux (Ernu, 2009: 205).

Le fait d'être devenu l'ennemi personnel de Staline, voire d'avoir tenté d'assassiner ce dernier n'empêche pas A. I. de déclarer que son adversaire fut «le seul de l'ancienne génération à avoir abordé de manière réaliste le projet soviétique» (Ernu, 2009: 203), en poursuivant et concrétisant le grand projet monarchiste de la Russie tzariste, après la Guerre Civile et une Première Guerre mondiale dévastatrice:

Le saut qualitatif accompli par l'Union Soviétique en cette période est stupéfiant. En une seule génération, nous sommes passés de l'esclavage à un degré raisonnable de civilisation, du labourage à cheval aux satellites. Dans ma classe, par exemple, nous étions tous des enfants d'anciens serfs de la glèbe, qui ne savaient même pas lire. Soixante ans plus tard, nous sommes devenus des intellectuels respectés partout dans le monde. Trois de mes camarades de classe comptent à leur actif le Prix Nobel. Un tel saut est unique dans l'histoire. Un saut qualitatif radical, d'une société semi-féodale à un super-pouvoir mondial, réalisé avec un énorme coût. C'est un prix trop important, car il s'agit de sacrifices humains. L'un de ces coût fut le Goulag. J'ai eu la «chance» d'y être envoyé, car mon projet n’a pas coïncidé avec

10 Relativisant quelque peu son propos ailleurs, A.I. déclare comme âge d'or (ou «dolce vita sovietica») l'époque brejnevienne, époque de relative opulence et de stabilité dans l'URSS: "Avec un minimum d'effort on obtenait l'essentiel nécessaire pour une vie décente: éducation, travail, conditions de vie, santé, logement, le strict nécessaire en produits de consommation» (Ernu, 2009: 174-176). Le personnage constate aussi que la pénurie peut être porteuse de plaisirs, les plaisirs étant mieux goûtés quand ils sont longuement recherchés et attendus. 
le grand projet stalinien. Je me suis opposé à ce projet, et je l'ai expié par quelques bonnes années de ma vie. (Ernu, 2009: 203)

Si A.I. admet sans réserve que le communisme a été "une authentique tragédie de l'histoire»(Ernu, 2009: 124), il a néanmoins des difficultés à relever les aspects décidément positifs du capitalisme et il s'étonne que le contemporain reste insensible aux sacrifices et autres rituels sanglants de notre époque. Indifférence qu'il met sur le compte de l'aliénation des individus, mais aussi sur l'Histoire elle-même, qui après s'être montrée comme tragédie (pendant l'époque stalinienne) se transforma par la suite en farce, ou en "Glamour Goulag» (ibid.) - dont nous vivons peut-être l'âge d'or. Mais, pour le spectateur vraiment lucide du temps présent, l'époque ne devrait pas être à la réjouissance:

Dans l'ancien Goulag nous avions de l'espoir car nous savions qu'il y avait une alternative, désormais nous ne pouvons plus nous permettre d'espoir, et la vraie tragédie, c'est que nous n'avons pas d'alternative. Or, ceci nous condamne à la chercher. (Ernu, 2009: 124)

\section{Pour conclure: Un écrivain «combattant pour les idées »}

Décidément, les idées de Vasile Ernu interpellent, car il relativise, voire renverse l'ordre dominant. Quoi qu'il en soit, il s'affiche volontiers en écrivain qui aime les idées, et profondément attaché à l'analyse de l'idéologique. Il se présente même en "combattant pour les idées» (Ernu, 2009: 140), qui s'adonne très librement à «l'exercice de la pensée» (ibid.) et qui se tient loin de tout jugement moral.

Si plus d'une fois, il lance des idées à risque, c'est, dit-il, pour ne pas voir sa pensée transformée en marchandise (Ernu, 2009: 76). Plus les thèmes abordés sont incommodes, plus il les adopte, car, pour lui, c'est le seul moyen d'ouvrir (et non pas de fermer) les débats (Ernu, Stănescu, 2010 : 122). Il considère sa tâche d'autant plus nécessaire qu'il écrit en Roumanie, là où les gens «ne croient en rien»(Ernu, 2009: 27) et où les littérateurs se livrent la plupart du temps à des exercices de style gratuits, qui ne les engagent aucunement.

Quand on lui demande quel est selon lui le plus grand écrivain de tous les temps, Vasile Ernu répond Gogol ${ }^{11}$, en qui il admire la capacité d'aller chercher des thématiques nouvelles là où personne n'avait fouillé auparavant, au lieu de se borner à imiter les grands écrivains de son temps

11 Il affirme au même endroit apprécier également Platonov et Bulgakov. 
(Ernu, Stănescu, 2010 : 44-49). Il admire donc Gogol - dans la lignée duquel Vasile Ernu compte bien s'inscrire - parce qu'il est un écrivain à idées, qui privilégie et développe celles-ci plus que le style ${ }^{12}$.

Enfin, Vasile Ernu est bien persuadé de se situer «à la frontière de l'Empire» (que celui-ci soit politique ou économique), dans un entre-deux, dans un «ni-ni», propre à «l'état devampire"13» (Ernu, 2009: 147-155). Et il vit cela comme une salutaire fatalité, qui lui laisse la possibilité de ne prendre aucune idée ni aucun fait pour des absolus intouchables, et de perpétuellement remettre en question ce qui est accepté de tous, notamment du «centre» (quel qu'il soit).

\section{Bibliographie}

Ernu V., [2006], 2010³, Născut în URSS, Avec une postface de Sorin Antohi, Iași, Ed. Polirom, coll. «Ego grafii».

Ernu V., Rogozanu C., Șiulea, C. et Țichindeleanu O., 2008, Iluzia anticomunismului. Lecturi critice ale Raportului Tismăneanu, Chișinău, Ed. Cartier.

Ernu V., 2009, Ultimii eretici ai Imperiului, Iași, Ed. Polirom, coll. «Ego grafii ».

Ernu V., Stănescu B.-A., 2010, Ceea ce ne desparte. Epistolarul de la Hanul lui Manuc, Préface de Radu Cosașu, Iași, Ed. Polirom, coll. «Duplex».

Ernu V., 2010, "Cum străinătatea a devenit noua mea Patrie», in Iancu, Bogdan, Prima mea călătorie în străinătate, Bucarest, Ed. Art, Série «Prima dată», p. 99-106.

Ernu V., 2012, Intelighenția rusă azi. Interviuri, discuții, polemici despre Rusia de ieri și de azi, Traduction de Vladimir Bulat, Chișinău, Ed. Cartier, coll. «Rotonda».

12 Le débat entre importance du style, respectivement de l'idéologie en littérature constitue le cœur du livre écrit à deux mains avec Bogdan-Alexandru Stănescu. Ce dernier considère que le plus grand écrivain de tous les temps est le maître du style James Joyce.

13 «L'état de vampire» est dans l'acception de Vasile Ernu une caractérisation culturelle imprimée par le centre "civilisé» aux êtres de la périphérie, que l'écrivain invite à ne pas se sentir lésés, mais de tirer, au contraire, un profit maximum de ce statut. 